\title{
Prevalencia de prescripción inapropiada de terapia supresora de ácido en adultos hospitalizados en un hospital general de Bogotá
}

\section{Prevalence of Inappropriate Prescription of Acid Suppression Therapy among Adults Hospitalized at a General Hospital in Bogotá}

\author{
Sebastián Posada Bustos, ${ }^{1}$ Nathalia De León Fernández, ${ }^{2}$ Rossmary González Morales, ${ }^{2}$ Jonathan Tihanyi Feldman, ${ }^{2}$ \\ José Fernando Vera Chamorro. ${ }^{3}$
}

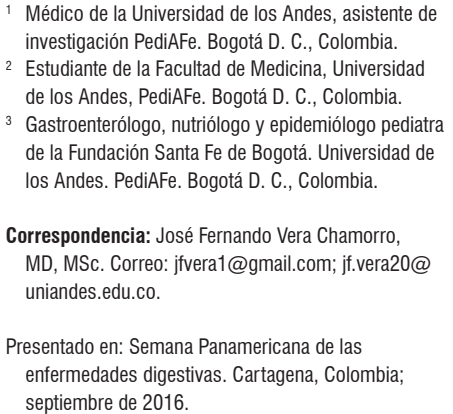

Correspondencia: José Fernando Vera Chamorro, MD, MSc. Correo: jfvera1@gmail.com; jf.vera20@ uniandes.edu.co.

Presentado en: Semana Panamericana de las enfermedades digestivas. Cartagena, Colombia; septiembre de 2016.

Fecha recibido: $01-08-17$ Fecha aceptado: 22-01-18

\begin{abstract}
Resumen
Objetivo: determinar la prevalencia de prescripción de terapia supresora de ácido (TSA) conformada por los inhibidores de la bomba de protones (IBP) y los antagonistas de los receptores $\mathrm{H}_{2}\left(\mathrm{AR}-\mathrm{H}_{2}\right)$ en pacientes adultos hospitalizados en el Hospital Universitario Fundación Santa Fe de Bogotá (HU-FSFB) que no corresponda con las indicaciones de las guías de práctica clínica (GPC). Métodos: estudio observacional descriptivo de corte transversal que incluyó pacientes adultos hospitalizados en el HU-FSFB con prescripción de TSA de novo, determinando las indicaciones de la TSA y comparándolas con las indicaciones validadas. Se excluyeron los pacientes con prescripción o consumo previo de TSA durante 2 semanas y pacientes internados en la unidad de cuidados intensivos $(\mathrm{UCl})$ o con hospitalización menor de un día. Resultados: entre enero y julio de 2015 se incluyeron 306 pacientes con un promedio de edad de 56,6 ( \pm 38$)$ años, con una prevalencia de prescripción de TSA sin indicación basada en la evidencia del 59,5\%. Las indicaciones no basadas en la evidencia más comunes fueron profilaxis de sangrado por úlcera gastrointestinal en pacientes de bajo riesgo $(64,9 \%)$ y causa no establecida $(13,7 \%)$. Se encontró una asociación estadísticamente significativa entre el tiempo de hospitalización menor de 7 días (odds ratio [OR]: 2,39; intervalo de confianza [IC] 95\%: 1,4-3,9) y edad menor de 60 años (OR: 1,9; IC 95\%: 1,2-3,03) con prescripción inapropiada de TSA. Conclusión: existe una alta prevalencia $(59,5 \%)$ de prescripción inapropiada de TSA en pacientes adultos hospitalizados con asociación positiva en menores de 60 años y corta hospitalización en el HU-FSFB.
\end{abstract}

Palabras clave

Inhibidores de la bomba de protones, hospitalizados, guías de práctica clínica, antagonistas de los receptores $\mathrm{H}_{2}$.

\begin{abstract}
Objective: This study's objective was to determine the prevalence of prescriptions of acid suppression therapy consisting of proton pump inhibitors (PPIs) or $\mathrm{H} 2$ receptor antagonists (H2RA) in adult patients hospitalized in the Hospital Universitario - Fundación Santa Fe de Bogotá (HU-FSFB - Santa Fe de Bogotá Foundation University Hospital) that are not in accordance with clinical practice guidelines (CPG). Methods: This is a cross-sectional descriptive observational study that included adult patients hospitalized in the HU-FSFB who were treated with acid suppressors for the first time. We determined the indications for prescriptions used and compared them with validated indications. We excluded patients who had previously taken acid suppressants for two weeks, patients admitted to the intensive care unit, and patients who stayed in the hospital for less than one day. Results: Between January and July 2015, 306 patients with an average age of 56.6 years were included in this study. The prevalence of acid suppression prescriptions without indications based on the evidence was $59.5 \%$. The most common indications were prophylaxis of bleeding due to gastrointestinal ulcers in low risk patients $(64.9 \%)$ and cause not established (13.7\%). Statistically significant associations were found between inappropriate prescription of acid suppression and hospitalization times of less than seven days (OR: $2.3995 \% \mathrm{Cl} 1.4-3.9$ ) and ages of less than 60 years (OR: $1.995 \% \mathrm{Cl} 1.2-3.03)$. Conclusion: The prevalence of inappropriate prescriptions of acid suppression for adult patients hospitalized in the HU-FSFB was (59.5\%). There were positive associations with ages under 60 years and short hospital stays.
\end{abstract}

Keywords

Proton pump inhibitors, hospitalized, clinical practice guidelines, $\mathrm{H}_{2}$ receptor antagonists 


\section{INTRODUCCIÓN}

La terapia supresora de ácido (TSA), conformada por los inhibidores de la bomba de protones (IBP) y los antagonistas de los receptores $\mathrm{H}_{2}\left(\mathrm{AR}-\mathrm{H}_{2}\right)$, es uno de los conjuntos de medicamentos con mayor prescripción en el mundo; su uso se ha considerado seguro debido a sus pocos efectos adversos (cefalea, dolor abdominal, náuseas, diarrea, vómito y flatulencia), lo que ha favorecido su uso indiscriminado en los últimos años (1). El mecanismo de acción de los IBP consiste en el bloqueo irreversible de la bomba $\mathrm{H}+/ \mathrm{K}+$ ATPasa (adenosina-trifosfatasa) en las células parietales y los $\mathrm{AR}-\mathrm{H}_{2}$ bloquean los receptores $\mathrm{H}_{2}$, lo que resulta en la reducción de la secreción de ácido (2).

Por una parte, estudios recientes motivados por el interés de evaluar las repercusiones de este aumento de la prescripción de la TSA han evidenciado que la administración de los IBP y AR- $\mathrm{H}_{2}$ está asociada con la disminución en la absorción de nutrientes como hierro, vitamina $\mathrm{B}_{12}$, calcio y magnesio (3-5). Por otra parte, el uso crónico de los IBP se ha asociado con el aumento en el riesgo de fracturas (6); y también existe una asociación positiva de la TSA con el aumento del riesgo de gastroenteritis, infecciones por Clostridium difficile y neumonía adquirida en la comunidad (7-10), y enfermedad renal crónica $(11,12)$.

En la literatura se ha reportado una prevalencia de prescripción de la TSA que no concuerda con las guías de práctica clínica (GPC) e indicaciones de la Food and Drug Administration (FDA); esta oscila entre el 50\% y $70 \%$ (13-23), lo que demuestra la alta tasa de sobreúso de estos medicamentos y el potencial riesgo de efectos adversos graves a los que se expone a los pacientes que reciben la TSA sin indicación.

Debido a que existen pocos reportes que evalúen el uso de TSA en Latinoamérica y Colombia, este estudio busca evaluar la prevalencia de prescripción no indicada en un hospital general de cuarto nivel en pacientes hospitalizados.

\section{MÉTODOS}

Estudio observacional descriptivo, de prevalencia y de corte transversal, de pacientes que cumplieron los siguientes criterios de inclusión: pacientes adultos hospitalizados en el Hospital Universitario Fundación Santa Fe de Bogotá (HU-FSFB) con prescripción de TSA de novo y que durante su estancia recibieron al menos una dosis de IBP (omeprazol, esomeprazol, pantoprazol o lansoprazol) o AR- $\mathrm{H}_{2}$ (ranitidina). Se determinaron las indicaciones de TSA y se compararon con las indicaciones validadas para establecer la prevalecía de prescripción inapropiada, las razones más frecuentes y la asociación entre variables clí- nicas con prescripción de TSA inapropiada. Los criterios de exclusión fueron: pacientes con prescripción o consumo previo de estos medicamentos durante al menos 2 semanas, y pacientes internados en unidad de cuidados intensivos (UCI) o con hospitalización menor de 1 día.

Para el tamaño de la muestra, se usó la fórmula de Kanh H. y Sempos C. (1989) para prevalencia puntual, en la que se estableció una población teórica mínima de 271 pacientes, con un error tipo I de 0,05 y una precisión del $90 \%$.

Los datos de las historias clínicas se recolectaron del sistema de información electrónico HIS-ISIS ${ }^{\circ}$ del HU-FSFB, teniendo en cuenta variables sociodemográficas y clínicas: antecedente de enfermedad ácido-péptica, medicamentos usados, diagnóstico principal de hospitalización, tiempo de hospitalización, tipo de TSA prescrito, dosis, duración de tratamiento, razón de uso de TSA y prescripción al egreso de TSA.

Para este estudio se usó un listado de indicaciones de prescripción de TSA en pacientes hospitalizados con base en las recomendaciones de la FDA, American Gastroenterology Association (AGA), American College of Gastroenterology (ACG) y American Society of Health-System Pharmacists (ASHP), previamente validadas y usadas en otros estudios $(19,22,24)$ (Tabla 1).

Tabla 1. Indicaciones de prescripción de TSA en pacientes hospitalizados según la evidencia $(19,22,24)^{*}$

\section{Indicaciones}

Tratamiento de mantenimiento de esofagitis erosiva
Tratamiento de ERGE sintomática
Tratamiento para la erradicación de la infección de Helicobacter pylori
Tratamiento de úlcera duodenal
Tratamiento de úlceras gástricas
Tratamiento del síndrome de Zollinger-Ellison
Tratamiento de úlcera gástrica inducida por los AINE
Sospecha de sangrado gastrointestinal alto
Dolor torácico con exámenes cardíacos y pulmonares negativos con
sospecha de causa debida a dispepsia o ERGE
Profilaxis del sangrado gastrointestinal por úlcera en:
Prevención de las úlceras gástricas inducidas por los AINE si hay:
Historia de enfermedad ulcerosa complicada
Uso concomitante de más de 1 AINE incluyendo aspirina
Uso de altas dosis de AINE
Uso concomitante de anticoagulantes
Historia de enfermedad ulcerosa péptica no complicada
Edad avanzada >65 años
Uso concomitante de esteroides
Pacientes intubados con coagulopatía
Pacientes quemados
Pacientes con injuria craneal severa

*FDA, AGA, ACG y ASHP. ERGE: enfermedad por reflujo gastroesofágico. 
Se realizó un análisis estadístico con medidas de estadística descriptiva de tendencia central y de dispersión, una prueba de chi-cuadrado $\left(\chi^{2}\right)$ junto con el cálculo de los odds ratio (OR) para establecer la asociación entre las variables de edad, sexo, tiempo de hospitalización y uso de medicamentos ulcerogénicos (antiinflamatorios no esteroideos [AINE], esteroides y anticoagulantes) con prescripción de TSA no basada en la evidencia, y tomando como un valor de $p<0,05$ como significativo. Se usó el programa estadístico STATA 12.0 para el análisis de los datos.

Este estudio fue aprobado por el comité de ética del HU-FSFB en la reunión del 14 de diciembre de 2015 y cumple con los criterios de buena práctica clínica y declaración de Helsinki 2013.

\section{RESULTADOS}

Se incluyeron 306 pacientes que cumplieron los criterios de inclusión y exclusión, entre enero a julio de 2015, con edad de 56,6 ( \pm 38$)$ años, $48 \%$ de sexo femenino, la mayoría con una formación superior al bachillerato en un $54 \%$, en su mayoría pertenecientes al régimen contributivo con medicina prepagada en un $70.4 \%$.

En cuanto a las variables clínicas, solo $42(13,7 \%)$ de los pacientes, tenían antecedente previo de enfermedad gastrointestinal, siendo la gastritis aguda o crónica la más común en $20(6,5 \%)$, enfermedad por reflujo gastroesofágico en 10 $(3,3 \%)$ y la úlcera gástrica o duodenal en 9 (3\%) pacientes.

Respecto al antecedente de uso de medicamentos ulcerogénicos, 28 pacientes $(9,1 \%)$ tenían prescrito anticoagulantes, 27 (8,8\%) AINE o aspirina y 17 (5,5\%), corticoides.

La media de duración de la hospitalización fue de 7,4 días, con mínimo de 1 día y máximo de 55 días. En cuanto al tipo de TSA utilizada, 266 prescripciones $(86,9 \%)$ se realizaron para los IBP; 28 (10,5\%) para los AR- $\mathrm{H}_{2}$ y 12 (4\%) para los 2 medicamentos.

Los medicamentos más usados en orden de frecuencia, según dosis y vía, fueron los siguientes: omeprazol $40 \mathrm{mg}$ cada 24 horas vía intravenosa (IV), omeprazol $20 \mathrm{mg}$ cada 24 horas vía oral (VO) y esomeprazol $20 \mathrm{mg}$ cada 24 horas VO; que corresponden a un $32,9 \%, 17 \%$ y $13 \%$ del total de prescripciones, respectivamente. Adicionalmente, la vía de administración más comúnmente empleada para el uso de la TSA fue la IV.

La prevalencia de prescripción inapropiada de TSA en los pacientes hospitalizados del HU-FSFB en el período de enero a julio de 2015 fue del 59,5\% (Figura 1).

En cuanto a las razones de prescripción de TSA basadas en la evidencia, en los 124 pacientes, la profilaxis del sangrado gastrointestinal por úlcera fue la más común en $67,7 \%$, seguida de la sospecha de sangrado gastrointestinal alto en un 10,5\% (Tabla 2).

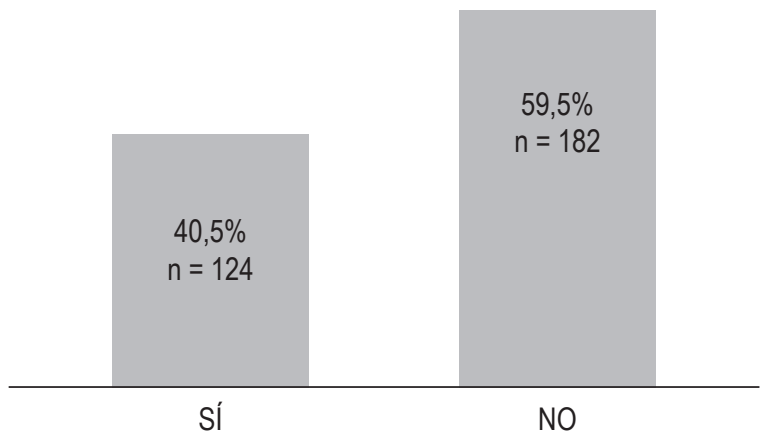

Figura 1. Prescripción de TSA de acuerdo con la evidencia $(n=306)$ $(19,22,26)$. Sí = basada en la evidencia; no = no basada en la evidencia. FDA, AGA, ACG y ASHP.

Tabla 2. Indicaciones de TSA basadas en la evidencia $(\mathrm{n}=124)$

\begin{tabular}{lcc}
\hline \multicolumn{1}{c}{ Indicación } & Pacientes & $\%$ \\
\hline Profilaxis del sangrado gastrointestinal por úlcera & 84 & 67,7 \\
Sospecha de sangrado gastrointestinal alto & 13 & 10,5 \\
Tratamiento de ERGE & 7 & 5,6 \\
Tratamiento de úlcera gástrica & 6 & 4,8 \\
Tratamiento de mantenimiento de esofagitis & 5 & 4,0 \\
erosiva & 3 & \\
Tratamiento de úlcera duodenal & 3 & 2,4 \\
Pacientes con injuria craneal severa & 3 & 2,4 \\
Dolor torácico con exámenes cardíacos y & & \\
pulmonares negativos con sospecha de & & \\
dispepsia o ERGE & & \\
\hline
\end{tabular}

Dentro de las razones de prescripción no basadas en la evidencia, en los 182 pacientes, las más comunes fueron: profilaxis de sangrado gastrointestinal en pacientes en postoperatorio de cirugía de bajo riesgo que no cumplen con los criterios establecidos de profilaxis (64,9\%), causa no establecida o no justificada en la historia clínica (13,7\%), síntomas dispépticos (pirosis y epigastralgia) $(10 \%)$ y prevención de sangrado gastrointestinal por úlcera en pacientes que no cumplen criterios (5,5\%) (Tabla 3).

Tabla 3. Indicación de TSA no basadas en la evidencia $(\mathrm{n}=182)$

\begin{tabular}{lcc}
\hline \multicolumn{1}{c}{ Indicación } & Pacientes & $\%$ \\
\hline Profilaxis de úlcera gástrica en pacientes en & 118 & 64,9 \\
postoperatorio & & \\
Causa no establecida & 25 & 13,7 \\
Síntomas dispépticos (pirosis y epigastralgia) & 18 & 9,9 \\
Prevención de úlcera gástrica, paciente que no & 10 & 5,5 \\
cumple los criterios & & \\
Historia de ERGE o úlcera no sintomática & 6 & 3,3 \\
Uso de sonda nasogástrica & 3 & 1,6 \\
Gastroenteritis & 2 & 1,1 \\
\hline
\end{tabular}


Del total de la muestra, 47 pacientes $(15,4 \%)$ egresaron del hospital con prescripción de TSA que no estaba basada en la evidencia.

Se encontró una asociación entre las variables de edad menor de 60 años (OR: 1,9; IC 95\%: 1,2-3,03) y hospitalización menor de 7 días (OR: 2,39; IC 95\%: 1,4-3,9) con prescripción inadecuada de TSA, datos estadísticamente significativos. No se encontró asociación con el sexo de los pacientes o uso de medicamentos ulcerogénicos (AINE, anticoagulante o corticoide), usando una prueba de $\chi^{2}$, con un valor de $\mathrm{p}$ significativo $<0,05$ (Tabla 4).

Tabla 4. Variables asociadas con prescripción inadecuada de la TSA

\begin{tabular}{llccc}
\hline \multicolumn{1}{c}{ Variable } & Categoría & OR & IC 95\% & Valor de $\mathbf{p}^{*}$ \\
\hline Edad & 18-60 años & 0,52 & $0,33-0,83$ & 0,006 \\
& $>60$ años & 1,91 & $1,20-3,03$ & 0,006 \\
Sexo & Masculino & 1,29 & $0,81-2,03$ & 0,282 \\
& Femenino & 0,78 & $0,49-1,23$ & 0,282 \\
Duración de la & $1-7$ días & 0,42 & $0,25-0,70$ & 0,001 \\
hospitalización & $>7$ días & 2,39 & $1,43-3,99$ & 0,001 \\
AINE & Sí & 1,65 & $0,75-3,65$ & 0,209 \\
& No & 0,6 & $0,27-1,33$ & 0,209 \\
Corticoide & Sí & 2,19 & $0,81-5,93$ & 0,114 \\
& No & 0,46 & $0,17-1,23$ & 0,114 \\
Anticoagulante & Sí & 1,3 & $0,60-2,85$ & 0,504 \\
& No & 0,77 & $0,35-1,67$ & 0,504 \\
\hline
\end{tabular}

*Prueba de $\chi^{2}$, valor significativo de $\mathrm{p}<0,05$.

\section{DISCUSIÓN}

La mayor parte de prescripciones de TSA en estos pacientes correspondió a los IBP, que guarda relación con respecto a la descrita en la literatura, en la que la prescripción de los IBP corresponde a un $76 \%$, frente a un $24 \%$ de los AR- $\mathrm{H}_{2}$ (13).

La prevalencia de prescripción de TSA no basada en la evidencia en la Fundación Santa Fe de Bogotá es del 59,5\%, que es significativamente alta. Sin embargo, dicha prevalencia está de acuerdo con otros estudios como los de Reidd y colaboradores, quienes encontraron que el 50\% de los pacientes del hospital de Colorado (Estados Unidos) no tenían una indicación válida para la prescripción de TSA (16); adicionalmente, Gupta y colaboradores reportaron un $73 \%$ de prevalencia de indicación inadecuada en el Centro Médico de la Universidad de Florida (19) y en otros centros como el hospital de Singapur se reportó un 55\% de uso no apropiado de TSA (20). En Perú, un estudio reportó que el $54,57 \%$ de prescripción de los IBP no estuvo basada en GPC (22). En un estudio realizado en el Hospital San Ignacio de Bogotá, la tasa de uso inadecuado fue del $59,7 \%$, muy similar a lo encontrado en este estudio (23). Otro estudio descrip- tivo que analizó más de 100000 prescripciones de los IBP en 2010 en Colombia, revisando los datos del sistema general de seguridad social en salud, encontró que el costo anual no justificado asciende a U. S. \$2 202590 (25). La prevalencia de prescripción inadecuada de TSA en el hospital está de acuerdo con las prevalencias reportadas en la literatura que oscilan entre el $50 \%$ y el $70 \%$, situación que se relaciona con costos elevados no justificados (13-23).

Dentro de las razones de prescripción de TSA basada en la evidencia en este estudio, la causa principal fue la profilaxis de sangrado gastrointestinal en pacientes con factores de riesgo, como el uso de los AINE, consistente con otros estudios como el de Bustamante y Scagliarini $(22,26)$.

En cuanto a las indicaciones no basadas en la evidencia, se encontró que la más frecuente es la profilaxis de sangrado gastrointestinal en pacientes de bajo riesgo, principalmente en postoperatorio, que no cumplen con los criterios de profilaxis, como el uso de los AINE; pacientes con injuria craneal, intubación o quemadura, que concuerdan con los hallazgos de otros estudios, cuyas frecuencias entre el $20 \%$ y el $30 \%$ muestran que la primera causa es la profilaxis de úlceras de estrés en pacientes de bajo riesgo $(19,22,24$, 26). Así mismo, en un estudio de los pacientes que habían recibido IBP realizado en el servicio de cirugía general del hospital de Lausanne, Suiza, el 79\% no tenía factores de riesgo para profilaxis de sangrado gastrointestinal, similar a nuestro estudio (21).

Cuando se revisa en la literatura la incidencia de sangrado gastrointestinal en pacientes de bajo riesgo, solamente un estudio en el que se evaluaron más de 78000 pacientes que no estaban en UCI encontró que la incidencia de sangrado evidente fue de $0,26 \%$ en pacientes que recibieron medicamentos antiácidos con respecto a $0,18 \%$ en pacientes sin medicamentos antiácidos, hecho que se traduce en un número necesario a tratar (NNT) de 834 pacientes para prevenir un episodio de sangrado gastrointestinal importante; lo que no es costo-efectivo $(14,27)$.

En este estudio no se encontró una asociación significativa entre la prescripción de la TSA con el uso de medicamentos ulcerogénicos (AINE, anticoagulantes y esteroides), con respecto a otros estudios como el de Gupta, en el que esta causa suma un 15\% del total (19); o el de Bustamante, en el que la polifarmacia fue la indicación más importante de prescripción de TSA inapropiada en el hospital de medicina interna (22); y el de Chia y colaboradores, en el que se presentó la asociación en el $35 \%$ de los pacientes (20).

Llama la atención que hasta a un $15 \%$ de los pacientes se les dio egreso con prescripción de TSA que fue prescrita sin evidencia, aunque menor con respecto a otros estudios como el de Ahrens en Alemania, donde un 58\% de 506 pacientes seguidos en 36 centros de atención primaria tenía prescripción de TSA sin indicación basada en la evidencia 
luego del egreso de hospitalización, lo que puede exponer a los pacientes al uso crónico de un medicamento, con efectos adversos y aumento de costos de manera innecesaria (28).

Por una parte, este estudio está sujeto a limitaciones como el sesgo de información que se encuentra en la revisión de historias clínicas, ya que no siempre se reportan todos los antecedentes o razones para tomar una conducta en el paciente, como la formulación de TSA. Por otra parte, este estudio se limita al servicio de hospitalización del hospital, excluyendo servicios importantes como consulta externa, en la que también se ha reportado sobreúso importante de estos medicamentos.

Este estudio advierte acerca de la elevada prevalencia de prescripción de TSA que no está basada en la evidencia en un hospital general en Colombia, mostrando la baja tasa de conocimiento y seguimiento de las indicaciones aprobadas de prescripción de la TSA. También hace evidente la necesidad de difundir las indicaciones aprobadas ante el personal médico con el fin de disminuir la tasa de indicaciones no apropiadas, el costo asociado y la exposición a riesgos innecesarios que pueden ser graves para los pacientes que reciben estos medicamentos. Por lo anterior sugerimos la creación de GPC adaptadas a nuestro medio que sean aplicables a todos los hospitales del país.

\section{Agradecimientos}

Al HU-FSFB y la Universidad de los Andes por su apoyo y compromiso con la investigación.

\section{REFERENCIAS}

1. Madanick RD. Proton pump inhibitor side effects and drug interactions: much ado about nothing? Cleve Clin J Med. 2011;78(1):39-49. DOI: 10.3949/ccjm.77a.10087.

2. Wallace JL, Sharkey KA. Pharmacotherapy of gastric acidity, peptic ulcers, and gastroesophageal reflux disease. En: Brunton LL, Chabner BA, Knollmann BC (editores). Goodman \& Gilman's the pharmacological basis of therapeutics. 12. ${ }^{\text {a }}$ edición. Nueva York: McGraw-Hill; 2011.pp. 1309-22.

3. Corleto VD, Festa S, Di Giulio E, et al. Proton pump inhibitor therapy and potential long-term harm. Curr Opin Endocrinol Diabetes Obes. 2014;21(1):3-8. DOI: 10.1097/ MED.0000000000000031.

4. Hess MW, Hoenderop JG, Bindels RJ, et al. Systematic review: hypomagnesaemia induced by proton pump inhibition. Aliment Pharmacol Ther. 2012;36(5):405-13. DOI: 10.1111/j.1365-2036.2012.05201.x.

5. ZipurskyJ, Macdonald EM, Hollands S, et al. Proton pump inhibitors and hospitalization with hypomagnesemia: a populationbased case-control study. PLoS Med. 2014;11(9):e1001736. DOI: $10.1371 /$ journal.pmed.1001736.
6. Yu EW, Bauer SR, Bain PA, et al. Proton pump inhibitors and risk of fractures: a meta-analysis of 11 international studies. Am J Med. 2011;124(6):519-26. DOI: 10.1016/j. amjmed.2011.01.007.

7. DeshpandeA, Pant C, PasupuletiV, etal.Association between proton pump inhibitor therapy and Clostridium difficile infection in a meta-analysis. Clin Gastroenterol Hepatol. 2012;10(3):225-33. DOI: 10.1016/j.cgh.2011.09.030.

8. KwokCS, ArthurAK, Anibueze CI, Singh S, Cavallazzi R, Loke YK. Risk of Clostridium difficile infection with acid suppressing drugs and antibiotics: meta-analysis. Am J Gastroenterol. 2012;107(7):1011-9. DOI: 10.1038/ajg.2012.108.

9. Eom CS, Jeon CY, Lim JW, Cho EG, Park SM, Lee KS. Use of acid-suppressive drugs and risk of pneumonia: a systematic review and meta-analysis. CMAJ. 2011;183(3):310-9. DOI: $10.1503 /$ cmaj.092129.

10. Azab M, Doo L, Doo DH, et al. Comparison of the hospitalacquired clostridium difficile infection risk of using proton pump inhibitors versus histamine- 2 receptor antagonists for prophylaxis and treatment of stress ulcers: a systematic review and meta-analysis. Gut Liver. 2017;11(6):781-788. DOI: $10.5009 /$ gnl16568.

11. Nochaiwong S, Ruengorn C, Awiphan R, et al. The association between proton pump inhibitor use and the risk of adverse kidney outcomes: a systematic review and metaanalysis. Nephrol Dial Transplant. 2018;33(2):331-342. DOI: $10.1093 / \mathrm{ndt} / \mathrm{gfw} 470$.

12. Wise J. Proton pump inhibitors are associated with risk of chronic kidney disease, study finds. BMJ. 2016;352:i128. DOI: $10.1136 /$ bmj.i128.

13. Barletta JF, Lat I, Micek ST, et al. Off-label use of gastrointestinal medications in the intensive care unit.J Intensive Care Med. 2015;30(4):217-25. DOI: 10.1177/0885066613516574.

14. Barletta JF, Sclar DA. Use of proton pump inhibitors for the provision of stress ulcer prophylaxis: clinical and economic consequences. Pharmacoeconomics. 2014;32(1):5-13. DOI: $10.1007 / \mathrm{s} 40273-013-0119-5$.

15. Farrell CP, Mercogliano G, Kuntz CL. Overuse of stress ulcer prophylaxis in the critical care setting and beyond. J Crit Care. 2010;25(2):214-20. DOI: 10.1016/j.jcrc.2009.05.014.

16. Reid M, Keniston A, Heller JC, et al. Inappropriate prescribing of proton pump inhibitors in hospitalized patients. J Hosp Med. 2012;7(5):421-5. DOI: 10.1002/jhm.1901.

17. Savarino V, Dulbecco P, de Bortoli N, et al. The appropriate use of proton pump inhibitors (PPIs): Need for a reappraisal. Eur J Intern Med. 2017;37:19-24. DOI: 10.1016/j. ejim.2016.10.007.

18. Thomas L, Culley EJ, Gladowski P, et al. Longitudinal analysis of the costs associated with inpatient initiation and subsequent outpatient continuation of proton pump inhibitor therapy for stress ulcer prophylaxis in a large managed care organization. J Manag Care Pharm. 2010;16(2):122-9. DOI: $10.18553 /$ jmcp.2010.16.2.122.

19. Gupta R, Garg P, Kottoor R, et al. Overuse of acid suppression therapyin hospitalized patients. South MedJ.2010;103(3):20711. DOI: 10.1097/SMJ.0b013e3181ce0e7a. 
20. Chia CT, Lim WP, Vu CK. Inappropriate use of proton pump inhibitors in a local setting. Singapore Med J. 2014;55(7):363-6. DOI: 10.11622/smedj.2014087.

21. Bez C, Perrottet N, Zingg T, et al. Stress ulcer prophylaxis in non-critically ill patients: a prospective evaluation of current practice in a general surgery department. J Eval Clin Pract. 2013;19(2):374-8. DOI: $10.1111 /$ j.13652753.2012.01838.x.

22. Bustamante Robles KY, Ticse Aguirre R, Cánepa Rondo IF, et al. Frequency of proton pump inhibitor prescription based in clinical practice guidelines in hospitalized patients in two academic hospitals in Lima, Peru. Rev Gastroenterol Peru. 2012;32(1):44-9.

23. Camacho R, Rodríguez A. Uso de los inhibidores de bomba de protones intravenosos en el Hospital Universitario de San Ignacio (HUSI). Universitas Médica. 2013;54(2):157-64.

24. Eid SM, Boueiz A, Paranji S, et al. Patterns and predictors of proton pump inhibitor overuse among academic and non- academic hospitalists. Intern Med. 2010;49(23):2561-8. DOI: 10.2169 /internalmedicine.49.4064.

25. Machado-AlbaJ,FernándezA, CastrillónJD, etal.Prescribing patterns and economic costs of proton pump inhibitors in Colombia. Colomb Med (Cali). 2013;44(1):13-8.

26. Scagliarini R, Magnani E, Praticò A, et al. Inadequate use of acid-suppressive therapy in hospitalized patients and its implications for general practice. Dig Dis Sci. 2005;50(12):230711. DOI: $10.1007 / \mathrm{s} 10620-005-3052-4$.

27. Herzig SJ, Vaughn BP, Howell MD, et al. Acid-suppressive medication use and the risk for nosocomial gastrointestinal tract bleeding. Arch Intern Med. 2011;171(11):991-7. DOI: $10.1001 /$ archinternmed.2011.14.

28. Ahrens D, Behrens G, Himmel W, et al. Appropriateness of proton pump inhibitor recommendations at hospital discharge and continuation in primary care. Int J Clin Pract. 2012;66(8):767773. DOI: $10.1111 /$ j.1742-1241.2012.02973.x. 